\title{
Thrombus versus Wall Biological Activities in Experimental Aortic Aneurysms
}

\author{
Michèle Coutard Ziad Touat Xavier Houard Anne Leclercq \\ Jean-Baptiste Michel \\ INSERM, U 698, and University Paris 7, Paris, France
}

\section{Key Words}

Aneurysm • Aneurysm, abdominal aortic · Proteases •

Intraluminal thrombus

\begin{abstract}
Background/Aim: The intraluminal thrombus (ILT) is considered to participate in abdominal aortic aneurysm (AAA) evolution. To assess whether this role proceeds via ILT influence on biological activity of the AAA wall, we studied the relationships between the levels of some relevant proteases and microparticles (MP) released by ILT versus wall in rat experimental AAAs. Methods and Results: Two weeks after elastase perfusion, ILT and AAA wall were incubated in cell culture medium and studies were performed on conditioned media. As shown by gelatin zymography, ILT released higher amounts of MMP9 than the wall, whereas the level of MMP2 activation (active/pro) was similar. Levels of elastase and urokinase plasminogen activator, plasmin and MPs, determined, respectively, by casein zymography, substrate hydrolysis and flow cytometry, were higher in ILT than in wall. Aneurysm diameter positively correlated with wall MMP9 levels, MMP2 activation, plasmin activity and MP release. Moreover, wall and ILT levels of pro- and active forms of MMP2, elastase and plasmin were positively correlated. Wall levels of MMP2 activation and plasmin activity also correlated with ILT
\end{abstract}

weight. Conclusion: The present data suggest that, in this experimental model, ILT may contribute to AAA evolution via its influence on the level of aneurysmal wall protease activity.

Copyright $\odot 2009$ S. Karger AG, Basel

\section{Introduction}

Abdominal aortic aneurysms (AAAs) are dilating lesions which result from extensive focal arterial remodeling where active proteolysis of the extracellular matrix plays a major role $[1,2]$. An intraluminal thrombus (ILT) of variable size and wall-covering surface is a common structural feature of human AAA and attention has been drawn to its potential role in aneurysmal evolution. Local hypoxia [3], wall thinning, increased inflammatory cell accumulation and cell apoptosis [4] occur in AAAs covered by a thrombus. The ILT appears to be a self-sustaining biologically active structure [5]. Renewal of its luminal pole, with platelet activation associated with microparticle (MP) production, was shown to participate in AAA evolution, as platelet inhibition prevented AAA enlargement both in man [6] and in a rat experimental model [7, 8]. Moreover, ILT of human AAAs has been shown to be a source of storage, release and activation of

Dr. Michèle Coutard

INSERM, U 698

Hôpital Bichat, Secteur Claude Bernard

46 rue Henri Huchard, FR-75877 Paris Cedex 18 (France)

Tel. +33 1402586 16, Fax +331402586 02,E-Mail michele.coutard@inserm.fr 
various proteases such as metalloproteinases (MMPs) [9, $10]$ and proteases of the fibrinolytic system $[10,11]$. In ILT of human AAAs, polymorphonuclear cells (PMNs) were previously shown to be the main source of MMP9 [9], whereas activated platelets may secrete MMP2 [12]. The high proteolytic activity of ILT provided an argument for its participation in the degradation of structural components of the arterial wall $[9,10]$. However, the expression and activity of matrix-degrading proteases were recently reported to be upregulated in walls of human AAAs exposed to flowing blood compared to walls covered by a thrombus [13]. The apparent discrepancy between these data and previous reports made the authors point out the need for further studies on the role of ILT-derived proteases in aneurysmal growth and the potential usefulness of an experimental model of AAA for this purpose.

Studies performed on human AAAs obtained at surgery have provided data of importance in describing AAA pathology, but these AAAs may be considered as 'end-point lesions'. Although in some aspects they represent 'acute lesions', experimental AAAs are induced in a given strain of animals under identical environmental conditions, and may be used to study mechanisms involved in the early phase of aneurysmal development. The elastase-induced model of AAA we described in 1990 in the rat [14] has been widely extended to various animal species and many different mechanisms have been reported to participate in the development of these aneurysms.

In the present study we used the rat model of elastaseinduced AAA to gain further insight into the role of ILT in AAA evolution via its biological activity such as protease release and MP production. Since the structure and permeability of the ILT have suggested its ability to convey macromolecules from the lumen to the underlying wall [5], the demonstration of relationships between protease levels in the ILT and the AAA wall may argue for a role of ILT biological activity in AAA evolution. We, thus, first studied levels of different proteases and MPs released by ILT and aneurysmal wall and then we analyzed possible correlations between them, and also between these levels and AAA size, an index of aneurysmal evolution.

\section{Methods}

\section{AAA Rat Model}

Lewis rats aged 10-12 weeks were from CEJ (Le Genest, France). To induce AAA, an isolated segment of abdominal aorta was perfused with 3 units of pancreatic porcine elastase (Sigma, E-1250 lot No. 083K7655) as previously described [14, 15].
The procedure and the animal care complied with the principles formulated by the National Society for Medical Research (animal facility agreement: No. B75-18-03), and this study was performed with the authorization (No. 75-101) for animal experimentation of the French Ministry of Agriculture.

\section{Measurement of Abdominal Aortic Diameter}

The diameter of the elastase-perfused abdominal aortic segment was measured in situ using a calibrated grid placed in the dissecting microscope eyepiece, postmortem for ruptured AAAs and at the time of sacrifice just before tissue sampling for the other rats.

As the aortic diameter increases by about $100 \%$ just after elastase perfusion (from 1.2 to $2.5 \mathrm{~mm}$ ), we considered the dilatation to be an AAA 2 weeks later (at the time of sacrifice) when an additional increase of $50 \%$ had occurred, i.e. an external diameter greater than $3.75 \mathrm{~mm}$.

For the study of the incidence of AAA and the relationship between AAA diameter and rupture, we collected data from different experiments, but not all these rats $(n=117)$ were used for the biochemical determinations described below.

\section{Sampling and Tissue Incubation}

Two weeks after elastase perfusion and under pentobarbital anesthesia, the abdominal aorta was exposed and the diameter of the elastase-perfused segment measured as described above. Aortic blood was rinsed by gentle perfusion of $\mathrm{NaCl} 9 \%$ via a catheter placed in the infrarenal aorta. Using a dissecting microscope, aortic segments were removed and AAAs opened longitudinally. The ILT was easily separated from the underlying wall using fine tweezers. Histological control of the method of separating ILT and wall was performed by analysis of paraffin sections of dissected ILT and wall, fixed with $3.7 \%$ formaldehyde. For each animal, aneurysmal ILT and underlying wall, and also nonaneurysmal elastase-perfused aortic wall (aortic diameter $\leq 3.75 \mathrm{~mm}$ ) and descending thoracic aorta, used as control perfused wall and control nonperfused walls, respectively, were dissected out, minced and incubated with serum-free RPMI-1640 culture medium (Gibco) at $37^{\circ} \mathrm{C}$ for $24 \mathrm{~h}$ in humidified $5 \% \mathrm{CO}_{2}$ air [16]. The different tissue samples were weighed (wet weight) and conditioned media from each sample were collected, centrifuged at $700 \mathrm{~g}$ and their protein concentration determined using a Bio-Rad protein assay. Samples were stored until use at $-80^{\circ} \mathrm{C}$. Ruptured AAAs were not used since rats died from hemorrhage and tissues were not suitable for enzymatic determination.

Blood of several rats was sampled, both before surgery and at sacrifice and for other rats only at sacrifice, on sodium citrate $(0.109 \mathrm{M})$ and centrifuged at $4^{\circ} \mathrm{C}$ at $700 \mathrm{~g}$ to obtain plasma.

\section{SDS-PAGE Zymography}

MMP and elastase activities were determined using gelatin and casein zymography, respectively, as previously described [1618]. A determined quantity of secreted proteins was loaded on gels. Proteolytic activities of tissue-conditioned media (appearing as white bands on gels stained with Coomassie blue) were quantified by densitometry using Scion image software and expressed per microgram of secreted protein in conditioned culture media. For control of MMP or serine protease activities, $20 \mathrm{mM}$ EDTA and $100 \mathrm{KIU} / \mathrm{ml}$ of aprotinin (Trasylol, Bayer) or $10 \mu \mathrm{M}$ of elastase inhibitor III (Calbiochem) or UPA-STOP ${ }^{\text {TM }}$ (American Diagnostica) were added to the incubation media. 
Fig. 1. a Ruptured AAA. b Diameter of ruptured $(\mathrm{n}=21)$ and intact $(\mathrm{n}=54)$ AAAs. ${ }^{*} \mathrm{p} \leq 0.002$ using the Mann-Whitney $\mathrm{U}$ test.
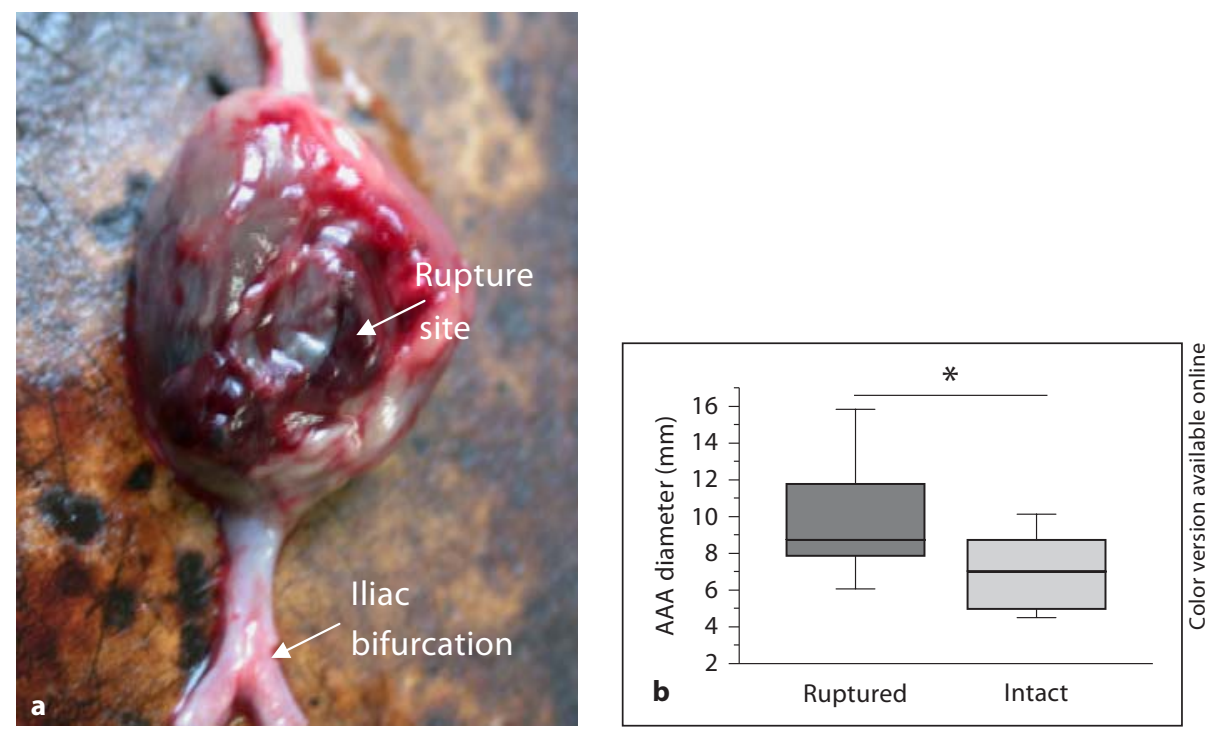

\section{Urokinase Plasminogen Activator Immunoblotting}

Proteins from ILT-conditioned media were resolved by SDSPAGE using $10 \%$ polyacrylamide gels and transferred onto nitrocellulose membranes (Amersham) incubated overnight at $4^{\circ} \mathrm{C}$ with $0.5 \mu \mathrm{g} / \mathrm{ml}$ urokinase plasminogen activator (uPA) antibody (human uPA B chain, American Diagnostica). After incubation with peroxidase-coupled secondary antibody, peroxidase activity was detected using chemiluminescence. uPA, pro-uPA and human urine (rich in uPA) were used as positive controls.

\section{Plasmin Activity}

Plasmin activity was determined by lysis of a specific substrate (Suc-Ala-Phe-Lys-AMC, Bachem). One to $4 \mu \mathrm{g}$ of secreted protein in tissue-conditioned media were added to $1 \mathrm{ml}$ buffer containing $50 \mathrm{~mm}$ Tris- $\mathrm{HCl}, 100 \mathrm{mM} \mathrm{NaCl}, 0.01 \%$ Tween 20 and the plasmin substrate at $40 \mu \mathrm{M}$ final concentration. Substrate hydrolysis was monitored every $10 \mathrm{~min}$ during $80 \mathrm{~min}$ by spectrofluorometry (Hitachi F-2000; $\lambda_{\mathrm{Exc}}=380 \mathrm{~nm}, \lambda_{\mathrm{Em}}=460 \mathrm{~nm}$ ). The rate of hydrolysis (released fluorescence per minute) was obtained and calculated per milligram of secreted protein.

\section{MP Determination by Flow Cytometry}

Cell-derived MPs from $500 \mu \mathrm{l}$ of plasma or tissue-conditioned media were isolated as described by Biro et al. [19] and were analyzed by flow cytometry (Coulter Epics XL with EXPO 32 software, Beckman Coulter) using annexin V-FITC labeling of surface-expressed phosphatidylserine. To set the background fluorescence, we used annexin $\mathrm{V}$ in the absence of calcium. A determined number of fluorescent beads were added to the samples before analysis to allow calculation of absolute values of MPs.

\section{Immunohistochemistry}

Five rats were used for histological studies.

The Apostain assay (Alexis Biochemicals) was used to localize apoptotic cells. Briefly, frozen sections were rapidly fixed with $4 \%$ paraformaldehyde followed by methanol/PBS. We, then applied, in the following order, $0.2 \%$ saponin, formamide:water $1: 1$ at $56^{\circ} \mathrm{C}, 3 \% \mathrm{H}_{2} \mathrm{O}_{2}, 3 \%$ casein, primary antibody $\mathrm{F} 7-2 \mathrm{~b}(5 \mu \mathrm{g} / \mathrm{ml}$ in $1 \%$ casein-PBS) and $3 \mu \mathrm{g} / \mathrm{ml}$ biotinylated goat anti-mouse II $^{\text {ry }}$ antibody (Zymed). HRP was revealed by Vectastain (Vector Labs). Native mouse IgG/A/M was used for negative control.

For uPA detection, acetone-fixed frozen sections were used and were applied in the following order: $3 \% \mathrm{H}_{2} \mathrm{O}_{2}, 10 \mu \mathrm{g} / \mathrm{ml} \mathrm{uPA}$ antibody (human uPA B chain, American Diagnostica) for 1 h 20 at room temperature, $5 \mu \mathrm{g} / \mathrm{ml}$ of biotinylated rat adsorbed antimouse IgG II ${ }^{\text {ry }}$ antibody (Vector Labs) and peroxidase-coupled streptavidin (Dako). Peroxidase was revealed by 3 '-diaminobenzidine tetrahydrochloride (Dako) and sections were counterstained with Mayer's hemalum.

Statistical Analysis

Nonparametric tests were used for the different statistical comparisons: the Kruskal-Wallis test for comparison of values between several groups, the Mann-Whitney U test for comparison between two groups, the Wilcoxon test for paired value comparison and the Spearman test for correlation analysis.

\section{Results}

\section{AAA Incidence, Diameter, Rupture and ILT Weight}

Out of a total of 117 rats which were successfully perfused with elastase, 42 rats (36\%) died before the time of sacrifice: 21 of these revealed AAA rupture (fig. 1a), the others showed aortic leakage or thrombosis very early after surgery or died from an undetermined cause and were excluded from the study. A total of 75 rats had developed AAA (21 ruptured, 54 intact) in the 2 weeks following perfusion. Lesion diameter was greater in ruptured than in intact AAAs (fig. 1b). 


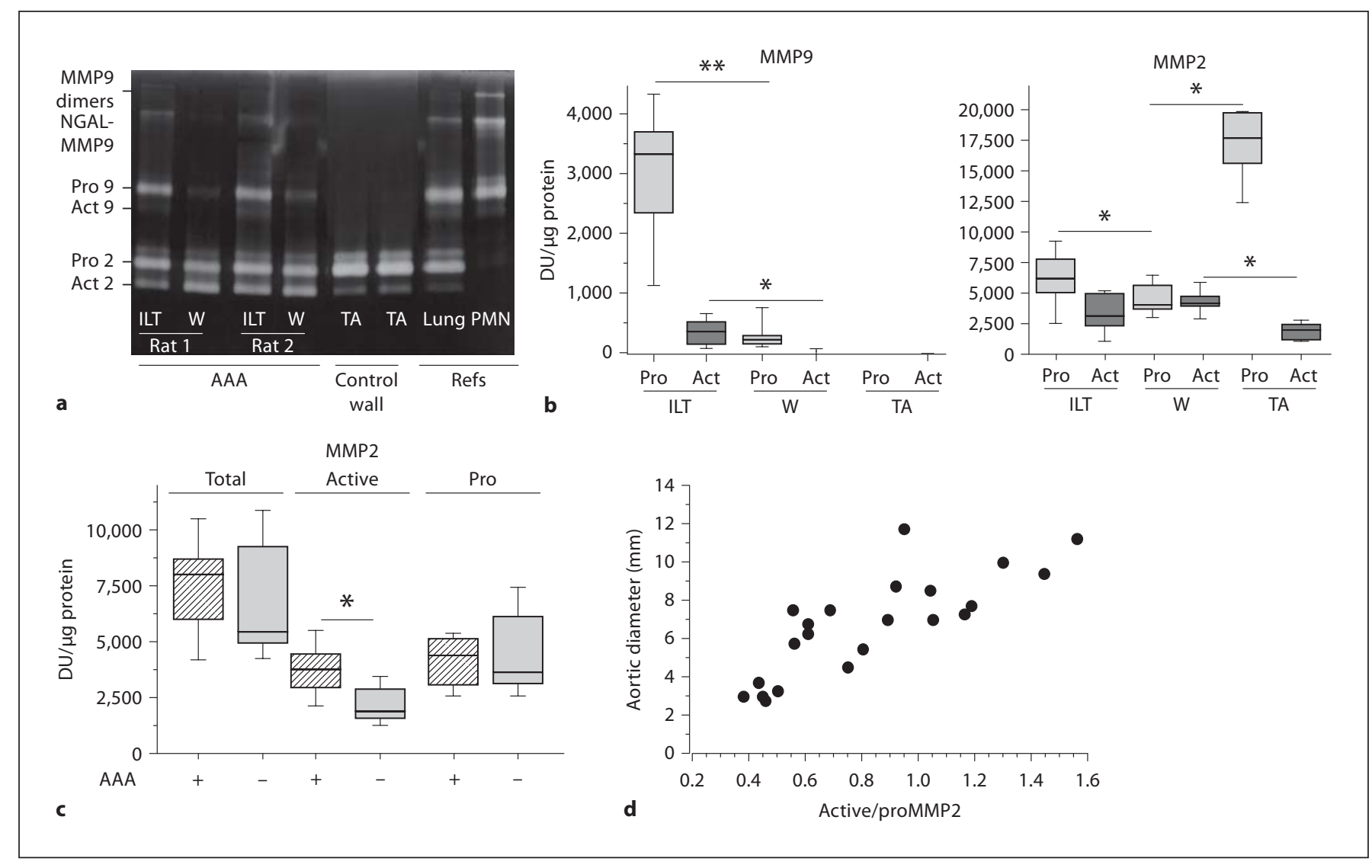

Fig. 2. MMP levels determined by SDS-PAGE gelatin zymography. a Zymogram obtained with media conditioned by ILT and AAA wall (7.5 $\mu$ g protein) and by control thoracic aortic wall (3.75 $\mu \mathrm{g}$ protein) from 2 Lewis rats, using rat lung-conditioned medium and supernatant of activated rat PMNs as references. b MMP9 and MMP2 levels in ILT, wall and thoracic aortic wall of rats with AAAs $(\mathrm{n}=9) .{ }^{*} \mathrm{p} \leq 0.05,{ }^{* *} \mathrm{p} \leq 0.01$ (using the Kruskal-Wallis test followed by the Wilcoxon paired test). c MMP2 levels in elastase-perfused aortic wall with $(+; \mathrm{n}=17)$ or without $(-; \mathrm{n}=6)$ AAA. ${ }^{*} \mathrm{p} \leq 0.05$ (using the Mann-Whitney U test). $\mathbf{d}$ Positive correlation (rho $=0.828, \mathrm{p}=0.0001$ by Spearman analysis) between active/proMMP2 levels of aortic wall and diameter of elastaseperfused aortic wall. $\mathrm{W}=$ Wall; $\mathrm{TA}=$ thoracic aortic wall; $\mathrm{DU}=$ densitometry units.
Forty-one AAAs were used to measure ILT weight and AAA diameter. Thirty-four AAAs contained an ILT (83\%), whereas 7 did not. The ILT weight correlated positively with the AAA diameter ( $r h o=0.673, \mathrm{p} \leq 0.0001$ by Spearman analysis). However, a few large AAAs ( $\mathrm{n}=$ 3 ; $\geq 8 \mathrm{~mm}$ ) had no or a very small ILT.

\section{MMP Levels}

In ILT- and Aortic Wall-Conditioned Media

As detected by zymography, MMP9 was mostly present in the proform and levels of pro- and active MMP9 were higher in ILT than in AAA wall; MMP9 was not observed in the wall of control thoracic aorta (fig. 2a, b). AAA diameter correlated positively with total MMP9 levels in AAA wall (rho $=0.507, \mathrm{p}=0.04)$. However, no significant differences were observed between MMP9 levels in aneurysm and nonaneurysmal elastase-perfused wall (data not shown). ProMMP2 was higher in the ILT than in AAA wall, whereas no significant difference in active MMP2 was observed. ProMMP2 levels were decreased in AAA wall compared to control thoracic aortic wall, whereas, in contrast, active MMP2 levels were significantly increased. In addition, active MMP2 was higher in AAA wall than in nonaneurysmal perfused aortic wall (fig. 2c). Furthermore, a positive correlation was found between aortic diameter and levels of MMP2 activation (active/proMMP2) in aortic wall when all elastaseperfused rats were considered (fig. 2d). This correlation persisted when only rats with AAA were considered $(\mathrm{rho}=0.653, \mathrm{p}=0.01)$. In addition, wall MMP2 activation 
(active/proMMP2) correlated positively with ILT weight in the AAA group $(\mathrm{rho}=0.648, \mathrm{p}=0.014)$. Moreover, active and total MMP2 levels in ILT correlated positively with their corresponding levels in AAA wall (table 1).

\section{In Plasma}

Plasma MMP9 levels at sacrifice were significantly increased ( $\mathrm{p}=0.036$ using paired Wilcoxon test) compared to values before surgery, whereas MMP2 levels had not changed (fig. 3a). This comparison was only performed in the AAA group. However, at 2 weeks, plasma levels of MMP9 and MMP2 were not different between elastase-perfused rats which had or had not developed an aneurysm (fig. 3b). No correlation was found between plasma MMP9 levels and thrombus weight or AAA diameter.

\section{Elastase Released by ILT and Aortic Wall}

Zymography in the presence of EDTA (an MMP inhibitor) (fig. 4a) showed a lytic band at about $30 \mathrm{kDa} \mathrm{MW}$ (similar to that of human neutrophil elastase: $28 \mathrm{kDa}$ ), and at a similar level as that obtained with supernatant of activated rat PMNs, known to secrete elastase. In addition, these lytic bands were greatly diminished in the presence of an elastase inhibitor during gel incubation (fig. 4a). These data converge to attribute this lytic activity to elastase. Elastase levels were higher in ILT than in wall (fig. 4b), and interestingly, a positive correlation was found between ILT and wall elastase levels (fig. 4c). In addition, within both ILT and wall, elastase levels correlated positively with MMP9 levels ( $\mathrm{p} \leq 0.05$ ). Moreover, ILT elastase levels also correlated positively with total MMP9 in AAA wall (table 1).

\section{uPA and Plasmin Activity Released by ILT and Aortic} Wall

Zymography gels incubated with an inhibitor of MMPs (EDTA) displayed another lytic band of about $50 \mathrm{kDa}$, which was of greater intensity in ILT than in AAA wallconditioned media (fig. 4a, b). This lytic band was still present in gels incubated with an elastase inhibitor and was abolished by aprotinin (a serine protease inhibitor; data not shown) or by uPA inhibitor (fig. 4a). uPA detection by immunoblotting (fig. $5 \mathrm{a}$ ) showed labeled bands at about 55 and $30 \mathrm{kDa}$ which may be, respectively, attributed to pro-uPA and to low-molecular-weight uPA. These bands were clearly more intense in ILT than in the wall. In addition, immunohistochemical localization of uPA showed labeling mainly associated with cells within the ILT (fig. 5b, c).

Proteases in Experimental Aneurysms
Table 1. Analysis of correlation between ILT and wall protease activities and between protease activities and AAA diameter

\begin{tabular}{|c|c|c|c|c|}
\hline \multicolumn{4}{|c|}{ Intraluminal thrombus } & \multirow{3}{*}{$\begin{array}{l}\text { AAA } \\
\text { diam- } \\
\text { eter }\end{array}$} \\
\hline \multirow{2}{*}{$\begin{array}{l}\text { MMP9 } \\
\text { total }\end{array}$} & \multicolumn{2}{|l|}{ MMP2 } & \multirow{2}{*}{$\begin{array}{l}\text { uPA elas- plas- } \\
\text { tase min }\end{array}$} & \\
\hline & $\begin{array}{l}\text { total ac- } \\
\text { tive }\end{array}$ & $\begin{array}{l}\text { Act/ } \\
\text { Pro }\end{array}$ & & \\
\hline
\end{tabular}

\begin{tabular}{llllllllll} 
AAA wall & & & & & & & & \\
MMP9 & & & & & & & & \\
$\quad$ Total & - & - & - & - & $\mathrm{N}^{*}$ & $\mathrm{P}^{*}$ & - & $\mathrm{P}^{*}$ \\
MMP2 & & & & & & & & \\
$\quad$ Total & - & $P^{*}$ & $\mathrm{P}^{* *}$ & - & - & - & - & - \\
$\quad$ Active & - & $\mathrm{P}^{*}$ & $P^{*}$ & - & - & - & - & - \\
Act/Pro & - & - & - & - & - & - & - & $\mathrm{P}^{* *}$ \\
uPA & - & - & - & - & - & $\mathrm{P}^{*}$ & - & - \\
Elastase & - & $\mathrm{N}^{*}$ & $\mathrm{~N}^{*}$ & - & - & $P^{*}$ & - & - \\
Plasmin & - & $\mathrm{N}^{*}$ & - & - & - & - & $P^{*}$ & $\mathrm{P}^{* *}$ \\
AAA diameter & - & - & - & - & - & - & - & - \\
\hline
\end{tabular}

${ }^{*} \mathrm{p} \leq 0.05,{ }^{* *} \mathrm{p} \leq 0.01$ statistically significant using Spearman test. $\mathrm{N}=$ Negative; $\mathrm{P}=$ positive; $-=$ no correlations. Italics represent positive correlations between ILT and wall levels of a given protease.

Plasmin activity (/mg of secreted protein) was higher in ILT than in AAA wall (fig. 5d), and these two activities were positively correlated ( $\mathrm{rho}=0.588, \mathrm{p}=0.03$; table 1). Plasmin activity was clearly increased in AAA wall compared to nonaneurysmal perfused wall and control aortic wall (fig. 5d). In addition, plasmin activity in AAA wall correlated positively with MMP2 activation (active/ proMMP2; rho $=0.494, \mathrm{p}=0.04$ ) and with AAA diameter (fig. 5e; table 1), whereas ILT plasmin activity did not. However, AAA wall plasmin activity correlated positively with ILT weight (rho $=0.491, \mathrm{p}=0.011)$ and also with total plasmin activity of the entire ILT with a high significance (rho $=0.900, \mathrm{p}<0.0001)$.

\section{Annexin V-Labeled Cell MPs}

Histologically, apoptotic cells, which are a source of cell MPs, were mainly observed within the intermediate layer of the ILT (fig. 6a) and also in different areas of AAA wall showing signs of inflammation.

\section{Plasma MPs}

MP levels were significantly increased at 2 weeks compared to before surgery only in elastase-perfused rats which had developed AAA. However, no significant differences were observed in rats with or without AAA (fig. 6b) partly due to a large variation in values. 


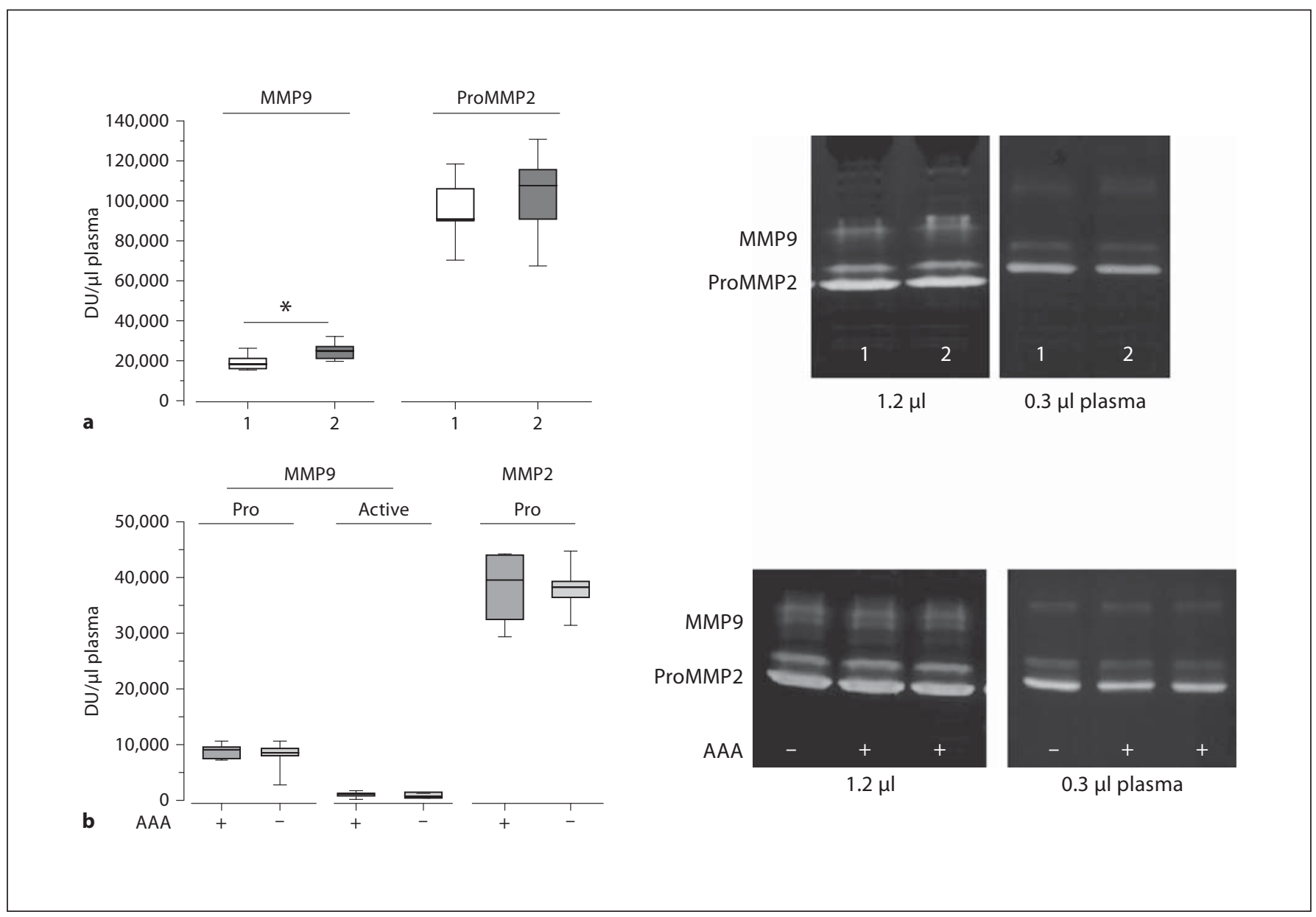

Fig. 3. Plasma MMPs determined by SDS-PAGE gelatin zymography. a Before elastase perfusion (1) and at 2 weeks $(2)$ measured only in the AAA group $(n=9)$. b At 2 weeks in elastase-perfused rats with $(+; n=16)$ or without $(-; \mathrm{n}=8)$ a developed AAA. DU $=$ Densitometry units.

MPs Released by ILT and Aortic Wall

A greater MP release (MP/mg tissue weight) was observed in ILT- than in wall-conditioned media. No difference in MP release was observed between AAA and nondilated elastase-perfused walls, although both levels were higher than that of control aortic wall (fig. 6c). Calculated numbers of MPs secreted by the entire tissue (total MPs) were not significantly different between ILT, AAA wall and nondilated wall, but all were higher than that of the control wall (fig. 6c). AAA diameter correlated with total MPs released by the ILT (rho $=0.437, \mathrm{p}=0.03$ ) but correlated more strongly with total MPs released by the AAA wall ( $\mathrm{rho}=0.545, \mathrm{p}=0.009$ ) (fig. $6 \mathrm{~d}$ ). No correlation was found between levels of MPs released by ILT and wall.

\section{Discussion}

In the present study, first we showed that, in rat experimental AAAs which develop rapidly, ILT weight correlated with AAA diameter. In human AAAs, it has also been reported that a rapid lesion expansion was associated with an increased ILT load [20]. However, in very few rats, large AAAs occurred with no or a very small-sized ILT. Similarly, in man, a small proportion of AAAs have no or only a small ILT. In the present study, the diameter of ruptured experimental AAAs was higher than that of intact ones, as previously reported for human AAAs [21], confirming that rapid growth and large diameter are risk factors for aneurysmal rupture [22]. In addition, a greater ILT volume has been reported in ruptured compared to 


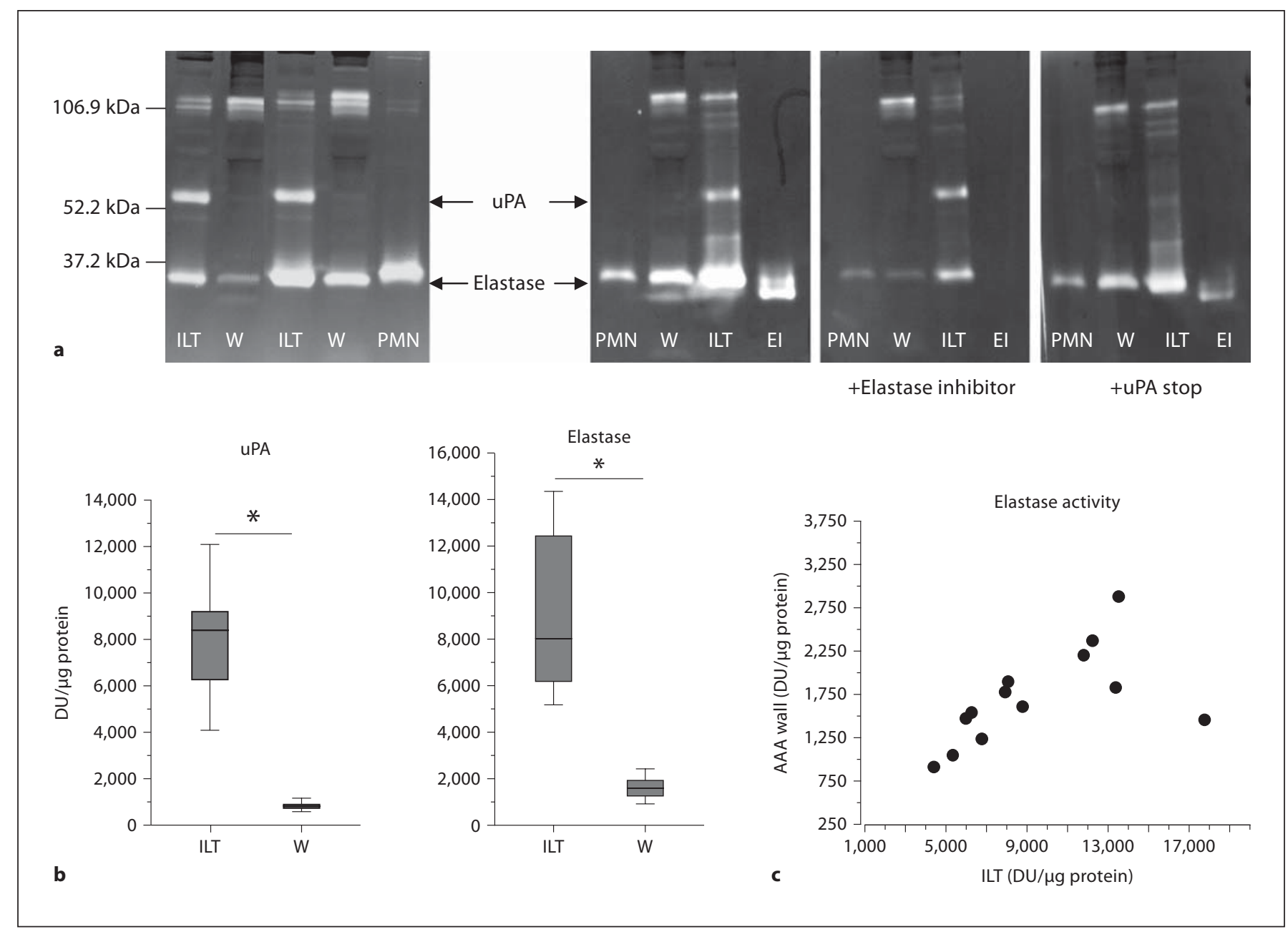

Fig. 4. SDS-PAGE casein zymography of aneurysmal ILT- and wall-conditioned media. a Zymograms obtained with conditioned media from ILT (5 $\mu$ g protein) and wall (20 $\mu \mathrm{g})$, supernatant of activated PMNs and human elastase (El), in the presence of EDTA (MMP inhibitor) alone or associated with inhibitors of elastase or uPA (uPA stop) during gel incubation. b uPA and elas- tase lytic activity of ILT- and wall-conditioned media $(\mathrm{n}=14)$. ${ }^{*} \mathrm{p} \leq 0.001$ (using the paired Wilcoxon test). c Positive correlation (rho $=0.670, \mathrm{p}=0.02$ by Spearman analysis) between elastase levels in ILT and in AAA wall. $\mathrm{W}=$ Wall; DU = densitometry units. intact human AAAs, although there was no difference in the ILT/AAA volume ratio [21].

The present data on MMPs in rat AAAs showed that, 2 weeks following elastase perfusion, in both ILT- and wall-conditioned media, total (active + pro) MMP2 levels were not different from those of control thoracic aorta, where smooth muscle cells constitutively secrete MMP2, whereas MMP9 levels were increased, as reported for these two MMPs in human AAA extracts [23]. In rat AAAs, ILT showed higher MMP9 levels than AAA wall, as reported in human AAAs $[9,23]$. The numerous PMNs present in rat ILT [15] probably accounted for a large part of the MMP9 levels in ILT, as shown for human AAAs [9], whereas MMP2 could originate from activated platelets [12]. In AAA wall, inflammatory cells (chiefly macrophages) may be the major source of MMP9 [24] whereas MMP2 could be produced by both macrophages and smooth muscle cells [25]. The involvement of various MMPs in human aneurysmal pathology is now well established [for recent reviews, see 26, 27]. Similarly, in the rat elastase AAA model, endogenous proteinases, including MMPs, are induced simultaneously with matrix destruction and inflammatory cell infiltration during the course of AAA development [28] and MMP inactivation 


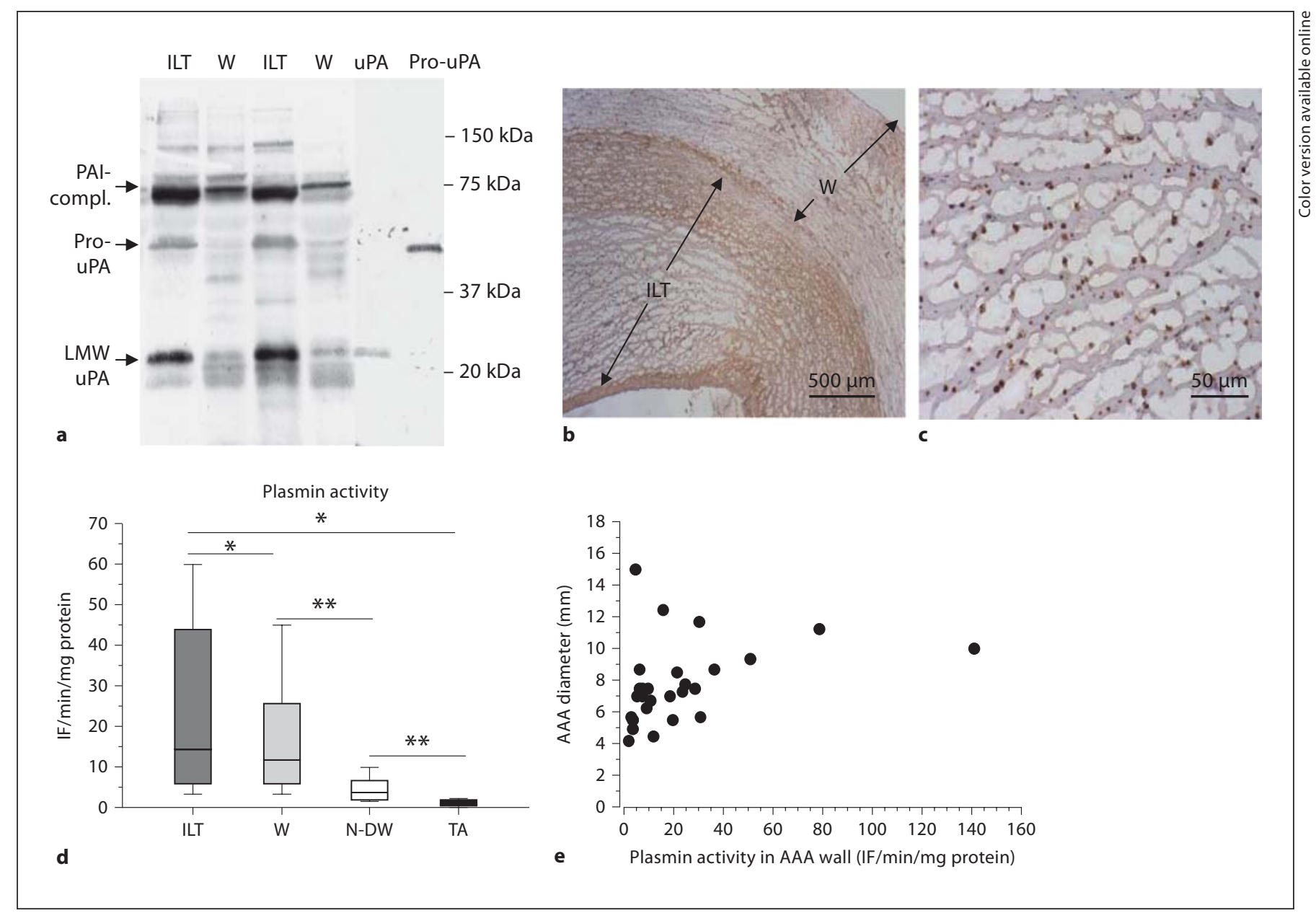

Fig. 5. a uPA immunoblot with aneurysmal ILT- and wall-conditioned media, and human uPA and pro-uPA as internal controls. $\mathrm{PAI}$-compl = Complexes of $\mathrm{u}-\mathrm{PA}$ and plasminogen activator inhibitor; LMW uPA = low-molecular-weight uPA. b, c uPA immunohistochemistry of AAA. Most of the labeling was located within the ILT (b) and labeling of cells in the middle part of ILT (c). d Plasmin activity in ILT- and wall-conditioned media of AAAs $(\mathrm{n}=28)$, nondilated perfused aortic wall $(\mathrm{N}-\mathrm{DW} ; \mathrm{n}=11)$ and control thoracic aortic (TA) wall $(\mathrm{n}=11)$ determined by hydrolysis rate of AFK-AMC substrate measured by intensity of fluorescence (IF) release $/ \mathrm{min} / \mathrm{mg}$ of secreted protein. ${ }^{*} \mathrm{p}<0.05$ significant differences between ILT and both wall and TA; ${ }^{* *} \mathrm{p}<0.01$ between $\mathrm{N}$-DW and TA (using the Kruskal-Wallis test followed by the Wilcoxon test), and between W and N-DW (using the Mann-Whitney $\mathrm{U}$ test). e Positive correlation ( $\mathrm{rho}=0.530, \mathrm{p}=0.006$ by Spearman analysis) between AAA diameter and plasmin activity in AAA wall-conditioned media $(n=29)$. $W=$ Wall. prevents AAA development $[29,30]$. In the present study, active MMP2 levels were increased both in ILT and wall of rat AAAs. Similarly, this MMP activation was observed in human AAA and correlated with the degree of inflammation [23]. Moreover, we showed that in elastaseperfused aortic segments, levels of MMP2 activation and of total MMP9 in AAA wall correlated with aortic diameter, suggesting a significant role for these MMPs during the early phase of AAA development. Recently, attention has been drawn to the role of MMP2 as an aneurysmal initiator [31] and, in human AAAs, MMP2 has also been shown to be the principal gelatinase present in small AAAs, whereas MMP9 activity is increased in large ones [32]. A role for MMP9 in experimental AAAs was shown by the suppression of AAA development by MMP9 gene disruption [33]. Present data on the study of relationships between ILT and wall levels of MMPs showed a positive correlation between ILT and wall levels of both pro- and active MMP2. A positive correlation was also found between ILT weight and the level of active MMP2. Together, 


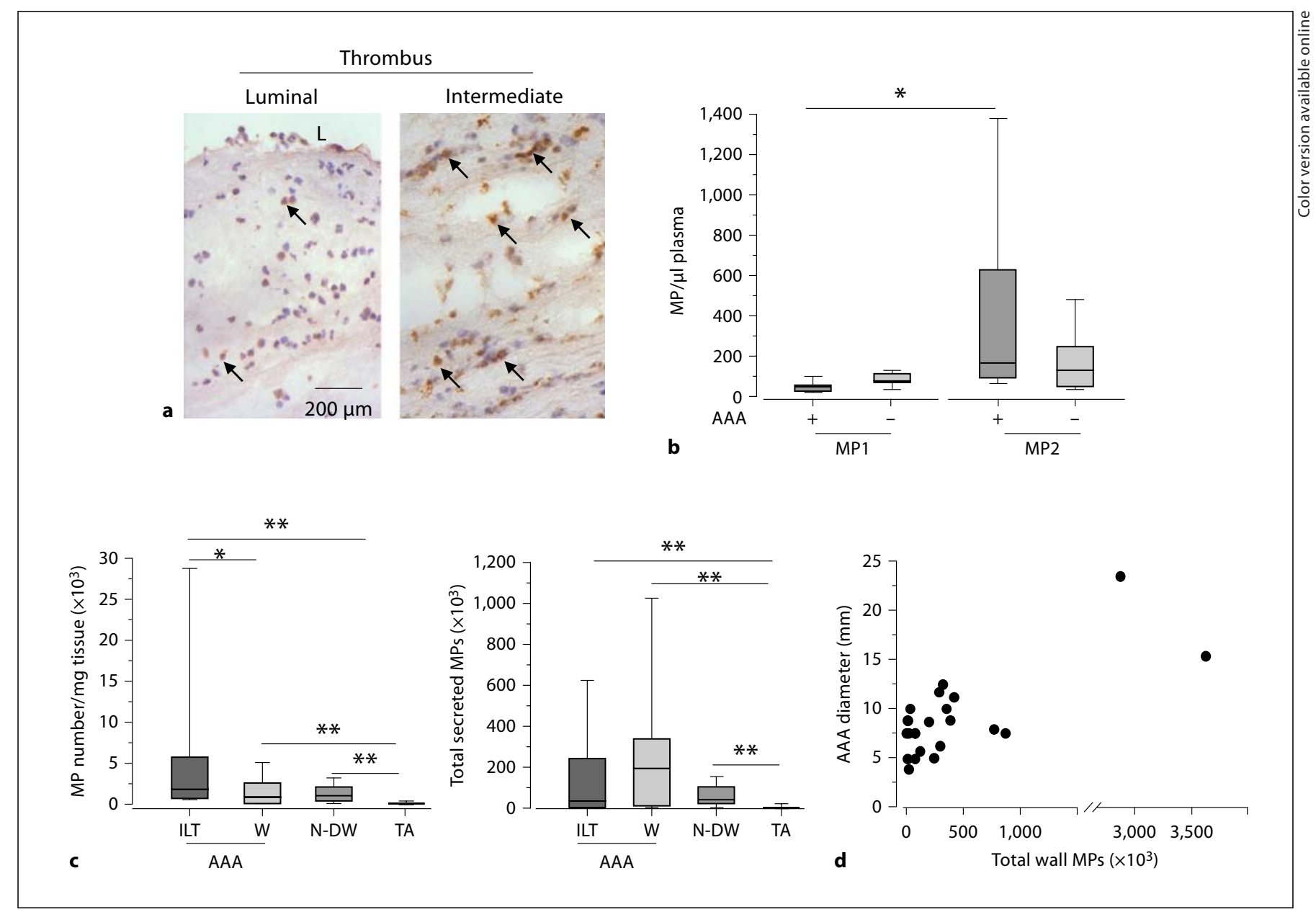

Fig. 6. a Apoptotic cells in the ILT labeled by the Apostain method. Labeling (arrows) was more intense in the middle part of ILT (right) than in the luminal part (left). $\mathrm{L}=$ Lumen. b-d Annexin V-labeled cell microparticles (MP) determined by flow cytometry. $\mathbf{b}$ In plasma before perfusion of elastase (MP1) and at sacrifice (MP2) in perfused rats with (+) or without $(-)$ AAA. ${ }^{*} \mathrm{p} \leq 0.05$ (using the paired Wilcoxon test). c In ILT- and wall-conditioned media of AAAs $(n=21)$, in conditioned media from nondilated perfused wall (N-DW; $\mathrm{n}=15)$ and control thoracic aorta (TA) wall $(\mathrm{n}=21) .{ }^{*} \mathrm{p} \leq 0.05,{ }^{* *} \mathrm{p} \leq 0.001$ (using the Kruskal-Wallis test followed by the paired Wilcoxon or Mann-Whitney $U$ tests). d Positive correlation ( $\mathrm{rho}=0.545, \mathrm{p}=0.009$ by Spearman analysis) between AAA diameter and total number of MPs secreted by AAA wall. $\mathrm{W}=$ Wall. these data suggest a link between MMP2 activity in ILT and AAA wall, the latter, as reported above, being itself related to AAA growth.

As for plasma MMP levels, increased MMP9 levels have been observed in AAA patients [34-36] although in only half of them by Hovsepian et al. [34]. Moreover, a decrease in plasma MMP9 and MMP3 levels after AAA exclusion [35] and an association between plasma MMP9 levels and AAA size and expansion [36] have been previously reported. In our study, at 2 weeks postelastase perfusion, MMP9 plasma levels were increased compared to levels before surgery (only studied in the AAA group).
However, no significant differences in MMP9 and MMP2 plasma levels were observed at the time of sacrifice between elastase-perfused rats with or without a developed AAA, showing that, in the early phases of rat experimental AAA formation, plasma MMP levels did not reflect the extent of AAA dilatation but rather the protease release consecutive to elastase perfusion.

Elastase was present in rat AAAs both in ILT and wall, but levels were higher in the former. Macrophages and, more importantly, PMNs are able to produce elastase and may account for the presence of this protease $[17,18]$, which cannot be due to residual porcine elastase, previ- 
ously shown to be undetectable $24 \mathrm{~h}$ postperfusion [28]. Levels of elastase and MMP9 were correlated both in ILT and wall suggesting a common cellular origin, probably from PMNs [18]. PMN apoptosis can be spontaneous and can also be induced by elastase or by activated macrophages which are present in the ILT of rat AAAs [15], and this may account for the numerous apoptotic cells observed in ILT. Besides its capacity to degrade different components of the extracellular matrix, neutrophil elastase has been shown to be involved in other important mechanisms of AAA development, such as MMP processing and also the impairment of cell recolonization of the AAA thrombus [17]. Elastase activity was previously shown to be increased in human AAAs compared to atherosclerotic lesions, and was highest in ruptured AAAs [37]. Moreover, the role of PMNs in AAA development has recently been emphasized by inhibition of experimental AAA formation by neutrophil depletion [38] or prevention of their recruitment $[39,40]$. However, in the present study no correlation was found between ILT or wall elastase levels and aneurysmal diameter; the very short half-life of PMNs activated by adhesion may account for the absence of such relationships. On the other hand, elastase levels in ILT and wall AAAs were correlated, as were MMP2 levels, reinforcing the evidence of relationships between ILT and wall biological activities.

The involvement of the fibrinolytic system in AAA pathology has been previously highlighted [41]. Plasmin generated by the action of plasminogen activators is able to degrade extracellular matrix components directly or indirectly by activating MMPs $[42,43]$. uPA is also able to stimulate MMP1 production via a plasmin-dependent pathway [44]. Recently, a critical role for uPA in the development of angiotensin II-induced AAAs has been demonstrated [45]. In rat AAAs, we showed that uPA was mainly present in the ILT and plasmin activity was also higher in ILT than in AAA wall. However, AAA diameter correlated with the level of plasmin activity in AAA wall. This latter also correlated with ILT weight and total plasmin activity of the entire ILT. This suggests that, rather than activity expressed as lytic activity/mg of secreted protein, the total ILT load of plasmin activity may be important in determining AAA wall plasmin activity and thus AAA evolution.

In the present study, MPs determined by the use of annexin $\mathrm{V}$ were released in larger numbers (/mg of tissue weight) by ILT than by the wall of rat AAAs. Previously, by in vivo scintigraphy of rat AAAs using ${ }^{99 \mathrm{~m}} \mathrm{Tc}$-annexin $\mathrm{V}$, we reported a preferential accumulation of annexin $\mathrm{V}$ in the platelet-rich luminal part of ILT [15]. In addi- tion, the numerous apoptotic cells observed in ILT of rat AAAs may also account for MP production. The present data also showed that AAA diameter correlated with total MPs secreted by the entire ILT, but it correlated more strongly with total MPs secreted by the AAA wall, suggesting that, in AAA, biological activity related to cell apoptosis and/or platelet activation is involved in lesion growth. Supporting these data, a lower expansion of AAAs by platelet inhibition in another rat model $[7,8]$ and higher plasma levels of MPs in AAA patients compared to healthy controls [7] have recently been reported. Accordingly, in our rat experiments and at 2 weeks following perfusion, mean plasma MP levels had increased in rats having developed an AAA whereas no significant difference was observed in rats with no dilatation. However, there was no significant difference between plasma MP levels at sacrifice in rats with or without AAAs. Since elastase perfusion increased wall MP levels even in perfused nondilated walls, these MPs could be released into the blood and may account for the lack of any significant difference, in addition to the large variation in values.

Data from this study were obtained from ILT- and AAA wall-conditioned media and thus only released proteins were considered. It should be borne in mind that molecules may be retained more or less within tissues due to differential binding to various structural components, such as fibrin, which binds latent MMP9 but not MMP2 [46] and which binds tPA more strongly than uPA [47]. Similar considerations also apply to molecules released by pathological tissues into the plasma. Moreover, the inverse proportion of blood leukocytes in the rat $(80 \%$ lymphocytes vs. $15 \%$ PMNs) compared to that in man (70\% PMNs vs. $15 \%$ lymphocytes) merits consideration when comparing rat and human studies. This is also the case for extrapolation of data obtained with this rat experimental model of AAA, where lesions develop rapidly after an acute aggression of the aortic wall by elastase perfusion, whereas human AAAs develop in a chronic manner often on an atherosclerotic aorta. In spite of these limitations, in the present study, we have shown first that dilatation of experimental AAAs was principally related to the levels of several relevant proteases and total MPs released by the AAA wall. Second, some of these AAA wall levels correlated with ILT size and/or with their ILT levels, indicating that the ILT may participate in AAA enlargement via its influence on relevant biological activities of the underlying wall, such as matrix-degrading protease activity. Thus, as possible clinical implications, the present results argue for the previously suggested pre- 
vention of thrombus formation to limit AAA expansion of small aneurysms [8]. Other clinical implications may be the development of in vivo imaging methods with molecular probes targeting levels of biological activity of the thrombus. Beside the involvement of the ILT in AAA expansion, it may be kept in mind that some authors by using computerized AAA models have suggested that ILT may also play a beneficial mechanical role in the risk of rupture by reducing AAA wall stress [48, 49]. Further studies are thus needed to define more precisely the relative importance of this dual role of the ILT in AAA rupture.

\section{Acknowledgments}

We are very grateful to Dr. Mary Osborne-Pellegrin for editing the manuscript. This study was supported by INSERM and the Leducq Foundation.

\section{References}

1 Thompson RW, Parks WC: Role of matrix metalloproteinases in abdominal aortic aneurysms. Ann NY Acad Sci 1996;800:157174.

-2 Sakalihasan N, Limet R, Defawe OD: Abdominal aortic aneurysm. Lancet 2005;365: 1577-1589.

-3 Vorp DA, Lee PC, Wang DH, Makaroun MS, Nemoto EM, Ogawa S, Webster MW: Association of intraluminal thrombus in abdominal aortic aneurysm with local hypoxia and wall weakening. J Vasc Surg 2001;34:291299.

4 Kazi M, Thyberg J, Religa P, Roy J, Eriksson P, Hedin U, Swedenborg J: Influence of intraluminal thrombus on structural and cellular composition of abdominal aortic aneurysm wall. J Vasc Surg 2003;38:1283-1292.

- 5 Adolph R, Vorp DA, Steed DL, Webster MW, Kameneva MW, Watkins SC: Cellular content and permeability of intraluminal thrombus in abdominal aortic aneurysms. J Vasc Surg 1997;25:916-926.

-6 Lindholt JS, Sorensen HT, Michel J-B, Thomsen HF, Henneberg EW: Low-dose aspirin may prevent growth and later surgical repair of medium-sized abdominal aortic aneurysms. Vasc Endovasc Surg 2008;42:329334.

7 Touat Z, Ollivier V, Dai J, Huisse M-G, Bezeaud A, Sebbag U, Palombi T, Rossignol P, Meilhac O, Guillin M-C, Michel J-B: Renewal of mural thrombus releases plasma marker and is involved in aortic abdominal aneurysm evolution. Am J Pathol 2006;168:10221030.

-8 Dai J, Louedec L, Philippe M, Michel J-B, Houard X: Effect of blocking platelet activation with AZD6140 on development of abdominal aortic aneurysm in a rat aneurys mal model. J Vasc Surg 2009;49:719-727.

$\checkmark 9$ Fontaine V, Jacob M-P, Houard X, Rossignol P, Plissonnier D, Angles-Cano E, Michel J-B: Involvement of the mural thrombus as a site of protease release and activation in human aortic aneurysms. Am J Pathol 2002;161: 1701-1710.
10 Carrell TW, Burnand KG, Booth NA, Humphries J, Smith A: Intraluminal thrombus enhances proteolysis in abdominal aortic aneurysms. Vascular 2006;14:9-16.

11 Houard X, Rouzet F, Touat Z, Philippe M, Dominguez M, Fontaine V, Sarda-Mantel L, Meulemans A, Le Guludec D, Meilhac O, Michel J-B: Topology of the fibrinolytic system within the mural thrombus of human abdominal aortic aneurysms. J Pathol 2007; 212:20-28.

12 Falcinelli E, Giannini S, Boschetti E, Gresele P: Platelets release active matrix metalloproteinase-2 in humans at a site of vascular injury: lack of inhibition by aspirin. Br J Haematol 2007;138:221-230.

13 Kazi M, Zhu C, Roy J, Paulsson-Berne G, Hamsten A, Swedenborg J, Hedin U, Eriksson P: Difference in matrix-degrading protease expression and activity between thrombus-free and thrombus-covered wall of abdominal aortic aneurysm. Arterioscler Thromb Vasc Biol 2005;25:1341-1346.

14 Anidjar S, Salzmann J-L, Gentric D, Lagneau P, Camilleri J-P, Michel J-B: Elastase-induced experimental aneurysms in rats. Circulation 1990;82:973-981.

15 Sarda-Mantel L, Coutard M, Rouzet F, Raguin $O$, Vrigneaud J-M, Hervatin F, Martet G, Touat Z, Merlet P, Le Guludec D, Michel $\mathrm{J}-\mathrm{B}:{ }^{99 \mathrm{~m}} \mathrm{Tc}-$ Annexin-V functional imaging of luminal thrombus activity in abdominal aortic aneurysms. Arterioscler Thromb Vasc Biol 2006;26:2153-2159.

16 Coutard M, Osborne-Pellegrin M, Fontaine V, Jacob M-P, Michel J-B: High flow induced arterial remodelling in rats with different susceptibilities to cerebral aneurysms. J Vasc Res 2006;43:217-228.

17 Fontaine V, Touat Z, Mtairag M, Vranckx R, Louedec L, Houard X, Andreassian B, Sebbag U, Palombi T, Jacob M-P, Meilhac O, Michel J-B: Role of leukocyte elastase in preventing cellular re-colonization of the mural thrombus. Am J Pathol 2004; 164:20772087.
18 Leclercq A, Houard X, Philippe M, Ollivier V, Sebbag U, Meilhac O, Michel J-B: Involvement of intraplaque hemorrhage in atherothrombosis evolution via neutrophil protease enrichment. J Leukoc Biol 2007;82: 1420-1429.

19 Biro E, Nieuwland R, Sturk A: Measuring circulating cell-derived microparticles. J Thromb Haemost 2004;2:1843-1844.

20 Wolf YG, Thomas WS, Brennan FJ, Goff WG, Sise MJ, Bernstein EF: Computed tomography scanning findings associated with rapid expansion of abdominal aortic aneurysms. J Vasc Surg 1994;20:529-535.

21 Hans SS, Jareunpoon O, Balasubramanian M, Zelenock GB: Size and location of thrombus in intact and ruptured abdominal aortic aneurysms. J Vasc Surg 2005;41:584-588.

22 Limet R, Sakalihasan N, Albert A: Determination of the expansion rate and incidence of rupture of abdominal aortic aneurysms. J Vasc Surg 1991;14:540-548.

23 Sakalihasan N, Delvenne P, Nusgens BV, Limet R, Lapiere CM: Activated forms of MMP2 and MMP9 in abdominal aortic aneurysms. J Vasc Surg 1996;24:127-133.

24 Thompson RW, Holmes DR, Mertens RA, Liao S, Botney MD, Mecham RP, Welgus HG, Parks WC: Production and localization of 92-kilodalton gelatinase in abdominal aortic aneurysms. An elastolytic metalloproteinase expressed by aneurysm-infiltrating macrophages. J Clin Invest 1995;96:318326.

25 Crowther M, Goodall S, Jones JL, Bell PRF, Thompson MM: Increased matrix metalloproteinase 2 expression in vascular smooth muscle cells cultured from abdominal aortic aneurysms. J Vasc Surg 2000;32:575-583.

26 Kadoglou NP, Liapis CD: Matrix metalloproteinases: contribution to pathogenesis, diagnosis, surveillance and treatment of abdominal aortic aneurysms. Curr Med Res Opin 2004;20:419-432.

27 Hobeika MJ, Thompson RW, Muhs BE, Brooks PC, Gagne PJ: Matrix metalloproteinases in peripheral vascular disease. J Vasc Surg 2007;45:849-857. 
-28 Halpern VJ, Nackman GB, Gandhi RH, Irizarry E, Scholes JV, Ramey WG, Tilson MD: The elastase infusion model of experimental aortic aneurysms: synchrony of induction of endogenous proteinases with matrix destruction and inflammatory cell response. J Vasc Surg 1994;20:51-60.

-29 Bigatel DA, Elmore JR, Carey DJ, CizmeciSmith G, Franklin DP, Youkey JR: The matrix metalloproteinase inhibitor BB-94 limits expansion of experimental aortic aneurysms. J Vasc Surg 1999;29:130-139.

-30 Moore G, Liao S, Curci JA, Starcher BC, Martin RL, Hendricks RT, Chen JJ, Thompson RW: Suppression of experimental aortic aneurysms by systemic treatment with a hydroxamate-based matrix metalloproteinase inhibitor. J Vasc Surg 1999;29:522-532.

-31 Thompson M, Cockerill G: Matrix metalloproteinase-2: the forgotten enzyme in aneurysm pathogenesis. Ann NY Acad Sci 2006; 1085:170-174.

-32 Freestone T, Turner RJ, Coady A, Higman DJ, Greenhalgh RM, Powell JT: Inflammation and matrix metalloproteinases in the enlarging abdominal aortic aneurysm. Arterioscler Thromb Vasc Biol 1995;15:11451151.

-33 Pyo R, Lee JK, Shipley JM, Curci JA, Mao D, Ziporin SJ, Ennis TL, Shapiro SD, Senior RM, Thompson RW: Targeted gene disruption of matrix metalloproteinase-9 (gelatinase B) suppresses development of experimental aortic aneurysms. J Clin Invest 2000; 105:1641-1649.

-34 Hovsepian DM, Ziporin SJ, Sakurai MK, Lee JK, Curci JA, Thompson RW: Elevated plasma levels of matrix metalloproteinase-9 in patients with abdominal aortic aneurysms: a circulating marker of degenerative aneurysm disease. J Vasc Inter Radiol 2000;11 1345-1352.
35 Sangiorgi G, D’Averio R, Mauriello A, Bondio M, Pontillo M, Castelvecchio S, Trimarchi S, Tolva V, Nano G, Rampoldi V, Spagnoli LG, Inglese L: Plasma levels of metalloproteinases- 3 and -9 as markers of successful abdominal aortic aneurysm exclusion after endovascular graft treatment. Circulation 2001;104:I288-I295.

36 Lindholt JS, Vammen S, Fasting H, Henneberg EW, Heickendorff L: The plasma level of matrix metalloproteinase 9 may predict the natural history of small abdominal aortic aneurysms. A preliminary study. Eur J Vasc Endovasc Surg 2000;20:281-285.

37 Cohen JR, Mandell C, Chang JB, Wise L: Elastin metabolism of the infrarenal aorta. J Vasc Surg 1988;7:210-214.

>38 Eliason JL, Hannawa KK, Ailawadi G, Sinha I, Ford JW, Deogracias MP, Roelofs KJ, Woodrum DT, Ennis TL, Henke PK, Stanley JC, Thompson RW: Neutrophil depletion inhibits experimental abdominal aortic aneurysm formation. Circulation 2005;112:232-240.

39 Hannawa KK, Eliason JL, Woodrum DT, Pearce CG, Roelofs KJ, Grigoryants V, Eagleton MJ, Henke PK, Wakefield TW, Myers DD, Stanley JC, Upchurch GR: L-Selectinmediated neutrophil recruitment in experimental rodent aneurysm formation. Circulation 2005;112:241-247.

40 Pagano MB, Bartoli MA, Ennis TL, Mao D, Simmons PM, Thompson RW, Pham CT: Critical role of dipeptidyl peptidase I in neutrophil recruitment during the development of experimental abdominal aortic aneurysms. Proc Natl Acad Sci USA 2007;104: 2855-2860.

41 Reilly JM: Plasminogen activators in abdominal aortic aneurysmal disease. Ann NY Acad Sci 1996;18:151-156.

42 Lijnen HR: Plasmin and matrix metalloproteinases in vascular remodeling. Thromb Haemost 2001;86:324-333.
43 Carmeliet P, Moons L, Lijnen R, Baes M, Lemaitre V, Tipping P, Drew A, Eeckout Y, Shapiro S, Lupu F, Collen D: Urokinase-generated plasmin activates matrix metalloproteinases during aneurysm formation. Nat Genet 1997;17:439-444

44 Zhang Y, Zhou Z-H, Bugge TH, Wahl LM: Urokinase-type plasminogen activator stimulation of monocyte matrix metalloproteinase- 1 production is mediated by plasmin dependent signaling through annexin A2 and inhibited by inactive plasmin. J Immunol 2007;179:3297-3304.

45 Deng GG, Martin-McNulty B, Sukovich DA, Freay A, Halks-Miller M, Thinnes T, Loskutoff DJ, Carmeliet P, Dole WP, Wang Y-X: Urokinase-type plasminogen activator plays a critical role in angiotensin II-induced abdominal aortic aneurysm. Circ Res 2003;92: 510-523.

46 Makowski GS, Rambsy ML: Binding of latent metalloproteinase 9 to fibrin: activation via a plasmin-dependent pathway. Inflammation 1998;22:287-305

47 Thorsen S, Glas-Greenwalt P, Astrup T: Differences in the binding to fibrin of urokinase and tissue plasminogen activator. Thromb Diath Haemorrh 1972;28:65-74.

48 Wang DH, Makaroun MS, Webster MW, Vorp DA: Effect of intraluminal thrombus on wall stress in patient-specific models of abdominal aortic aneurysm. J Vasc Surg 2002;36:598-604.

49 Bluestein D, Dumont K, De Beule M, Ricotta J, Impellizzeri P, Verhegghe B, Verdonck P: Intraluminal thrombus and risk of rupture in patient specific abdominal aortic aneurysm - FSI modelling. Comput Methods Biomech Biomed Engin 2009;12:73-81. 